\title{
Prostaglandin F2 receptor inhibitor (PTGFRN) a cell adhesion molecule, promotes cell growth and migration in glioblastoma
}

\section{Uchurappa Mala}

Indian Institute of Science Bangalore

\section{Tapan Kumar Baral}

Indian Institute of Science Bangalore

Kumaravel Somasundaram ( $\sim$ skumar1@iisc.ac.in )

Indian Institute of Science Bangalore

\section{Research Article}

Keywords: Glioblastoma, CAM, PTGFRN, GSC, Growth, Migration

Posted Date: March 12th, 2021

DOI: https://doi.org/10.21203/rs.3.rs-258939/v1

License: (c) (i) This work is licensed under a Creative Commons Attribution 4.0 International License.

Read Full License 


\section{Abstract \\ Background}

Glioblastoma (GBM) is the most common primary malignant brain tumor in adults exhibiting infiltration into surrounding tissues, recurrence, and resistance to therapy. GBM infiltration is accomplished by many deregulated factors such as cell adhesion molecules (CAMs), which are membrane proteins that participate in cell-cell and cell-ECM interactions to regulate survival, proliferation, migration, and stemness.

\section{Methods}

A comprehensive bioinformatics analysis of CAMs $(n=518)$ in multiple available datasets revealed genetic and epigenetic alterations among CAMs in GBM. Univariate Cox regression analysis using TCGA dataset identified 127 CAMs to be significantly correlated with survival. The poor prognostic indicator PTGFRN was chosen to study its role in glioma. Silencing of PTGFRN in glioma cell lines was achieved by stable expression of short hairpin RNA (shRNA) against PTGFRN gene. PTGFRN was silenced and performed cell growth, migration, invasion, cell cycle, and apoptosis assays were performed. Neurosphere and limiting delusion assays were also performed after silencing of PTGFRN to know its role in GSCs.

\section{Results}

Among the differentially regulated CAMs ( $n=181,34.9 \%)$, major proportion of them were found to be regulated by miRNAs $(n=95,49.7 \%)$ followed by DNA methylation $(n=32,16.7 \%)$, and gene copy number variation ( $n=12,6.2 \%)$. We found that PTGFRN (Prostaglandin F2 receptor inhibitor) to be upregulated in GBM tumor samples and cell lines with a significant poor prognostic correlation with patient survival. Silencing PTGFRN diminished cell growth, colony formation, anchorage-independent growth, migration, and invasion and also led to cell cycle arrest and induction of apoptosis. At mechanistic level, silencing of PTGFRN reduced pro-proliferative and promigratory signaling pathways such as ERK, AKT, and mTOR. PTGFRN upregulation was found to be due to loss of its promoter methylation and downregulation of miR-137 in GBM. PTGFRN was also found to be higher in glioma stem-like cells (GSCs) and is required for GSC growth and survival.

\section{Conclusion}

In this study, we provide a comprehensive overview of the differential regulation of CAMs and causes for their deregulation. We also establish an oncogenic role of PTGFRN in GBM thus signifying it as a potential therapeutic target. 


\section{Background}

Glioblastoma (GBM) is the most common primary malignant tumor of brain neoplasia in adults and GBM patients confer worst prognosis with the median survival of around one year (1). GBM cells infiltrate into the brain parenchyma and are responsible for the complications encountered in surgery, therapy, and the lethality of the disease (2).

Adhesion of cells to a substratum (ECM) and to one another is primarily accomplished by a family of surface proteins called cell adhesion molecules (CAMs), which are involved in regulation of normal development and pathology of diseases including cancer (3). Initially, CAMs were depicted as tumor suppressors; however, many reports emphasized their oncogenic functions in several cancers including glioma $(4,5)$.

Prostaglandin F2 receptor inhibitor (PTGFRN) is a type I (single pass) transmembrane Ig superfamily CAM which was shown to be upregulated in several cancers including glioma (6-8). PTGFRN exhibit gene fusion (PTGFRN-NOTCH2) in colorectal cancer (8) and point mutations in small cell lung cancer (9). It interacts with tetraspanins (CD9 and CD81) (10), integrins (11), Ezrin-Radixin-Moesin (ERM) proteins (12), and $\gamma$-secretase (13) to regulate cell adhesion and migration. It was also found to be involved in adipocyte maturation (14), muscle regeneration (15), tumor angiogenesis, metastasis (16), and pathogen infection (17). Recently, PTGFRN has been shown to be overexpressed in GBM, promoting cell growth and resistance to radiation via PI3K-AKT signaling (18). However, its role in migration, invasion, and regulation of its expression in GBM is not known.

Though, several reports emphasize the importance of individual CAMs in GBM initiation and progression, none of them attempted to overview all the CAMs. In the present study, we analysed 518 CAMs for their transcriptional changes in GBM using multiple datasets and explored the probable causes for the deregulation. Further, we also provide experimental evidence to establish the pro-proliferative, promigratory function, and regulation of PTGFRN, an upregulated CAM, in GBM.

\section{Materials And Methods}

\section{Compilation of CAMs}

A list of manually curated 518 CAMs (Supplementary Table 1) was prepared from various sources such as Entrez query 'CAMs and homo sapiens', GO term annotations related to cell adhesion and literature was used for analysis in this study.

\section{Differential expression analysis}

The gene expression data for GBM was downloaded from TCGA, REMBRANDT, GSE7696, and GSE22866. The differential expression was calculated by subtracting average value of control samples from average of GBM samples. Statistical significance was tested using Wilcox Mann-Whitney test. Genes showing 
fold change $\leq-0.58$ or $\geq 0.58$ and significant $p$-value ( $p$-value $\leq 0.05$ ) were considered as differentially expressed.

\section{Survival Analysis}

All CAMs were subjected for univariate cox regression analysis using TCGA Agilent dataset and SPSS software. GraphPad Prism software 5.0 was used for the Kaplan Meier survival analysis.

\section{Copy number variation}

Copy number variation of CAMs was analysed using data from cBioPortal (http://www.cbioportal.org/) and calculated the percentage of samples in which a particular CAM amplified or deleted.

\section{Methylation data analysis}

The CpGs corresponding to differentially expressed genes were fetched from TCGA DNA Methylation dataset (Illumina Infinium Human DNA methylation 450K array). The data for control samples was taken from GSE79122. For each probe, the differential beta value was calculated by subtracting the average beta value in control from the average beta value in the GBM. In order to identify differentially methylated probes, the difference of $>0.3$ absolute beta value. Wilcox Mann-Whitney test was used to calculate statistical significance.

\section{MicroRNA}

The differentially regulated CAMs were used as an input for miRwalk. The miRNAs which were predicted by minimum, 7 or more algorithms to target the CAMs were taken to further analysis. The miRNAs that were reciprocally regulated with respect to their target CAMs were considered as miRNA and CAM pairs.

\section{Cell lines, normal brain tissues, and plasmids}

Glioma cell lines (U373, T98G, U251, U87, LN229, U343, LN18, A172, and U138), immortalized astrocytes (NHA and SVG), and 293T were cultured in DMEM (Sigma-Aldrich, \#D5648) supplemented with 10\% Fetal Bovine Serum (FBS), Penicillin, and Streptomycin. U251, U87, U373, T98G, and 293T were bought from ECACC. LN229 and NHA were gifted from late Dr. Abhijit Guha (University of Toronto, Canada). Patient tumor derived primary GSC lines MGG8, MGG6, and MGG4 were procured from Dr. Wakimoto $H$. (Massachusetts General Hospital, Boston, USA) and 1035 was obtained from Dr. Santosh Kesari (University of California, San Diego, USA) and were cultured as neurospheres.

Non-tumorous control brain tissue samples (N1-N5) procured from patients with intractable epilepsy during surgery at National Institute of Mental Health and Sciences (NIMHANS), Bangalore, India. The tissues were snap-frozen in liquid nitrogen and stored at $-80^{\circ} \mathrm{C}$ and used for RNA isolation. The tissue samples were obtained after informed and taken written consent from all patients prior to use in the study. The study has been approved by the ethics committee of the NIMHANS and Indian Institute of 
Science (IISc). In the present study control brain tissue samples used for isolation of RNA and to measure PTGFRN transcript levels.

PTGFRN shRNAs TRCN00000057448 to 57452 (Sigma-Aldrich) were used for silencing PTGFRN and pcDNA3.2/V5 mmu-miR-137 (Addgene) was used for overexpression of miR-137.

\section{Lentivirus preparation and transduction}

293T cells transfected with shRNA $(4 \mu \mathrm{g})$ along with the helper plasmids pSPAX and pMD2.G (3:1) using Lipofectamine 2000 (Invitrogen, \#11668019) transfection reagent in $60 \mathrm{~mm}$ dish. The media was changed after $5 \mathrm{~h}$ of transfection and supernatant, which contain virus particles, was collected after $60 \mathrm{~h}$ of transfection and the virus was used to infect glioma cells in presence of $8 \mu \mathrm{g} / \mathrm{ml}$ of Polybrene (Sigma, \#107689).

\section{CDNA conversion and qPCR}

The total RNA was isolated by using Trizol (Sigma-Aldrich, \#T9424) method. RNA was converted to cDNA by using cDNA conversion kit (Life Technologies, \#4368813). Subsequently, RT-qPCR was performed and fold change was calculated by $\Delta \Delta$ ct method.

\section{Western blotting}

The RIPA buffer was used to lyse the cells and supernatant was collected. Protein was quantified using Bradford reagent and the required amount of protein was resolved in the SDS-PAGE gel and performed Western blotting. The primary antibodies anti-PTGFRN (ab97567, abcam), anti-phospho-ERK1/2 (\#9101, CST), anti-ERK (\#9102, CST), anti-phospho-AKT (\#9271, CST), anti-AKT (\#4691, CST), anti-phosphop70S6 (\#9208, CST), anti-p70S6 (\#2708, CST), anti-phospho-4EBP1 (\#9456, CST), anti-4EBP1 (\#9452, CST) and anti- $\beta$-actin (\#A3854, Sigma-Aldrich) were used in this study.

\section{Proliferation assay}

The cell viability was assessed by trypan blue assay. Briefly, the cells expressing control shRNA or shRNA against PTGFRN were plated in 6 well plate, $10^{4}$ cells per well. Cell viability checked on every $3^{\text {rd }}$ day using Vi-cell counter (Beckman Coulter, \#383722) and normalization was done using the reading of day 1 as control for each condition. Statistical analysis was done using Student's t-test.

\section{Colony suppression assay}

The cells stably expressing shRNA were counted and plated in a 6 well plate in triplicates at the seeding density of $10^{3}$ cells/well and grown for 2-3 weeks replacing the media every 2-3 days once. Colonies were fixed in chilled methanol overnight followed by staining with crystal violet $(0.05 \% \mathrm{w} / \mathrm{v})$ for $30 \mathrm{~min}$. Colonies were quantified either by manual counting or counting using ImageJ software. The statistical significance was calculated by Student's t-test. 


\section{Soft agar colony formation assay}

In the soft agar assay, cells were counted and plated in $10^{4}$ cells/well in $1.2 \mathrm{ml}$ of $0.4 \%$ of low melting agarose (BD Biosciences, \#214230) in 6 well plate containing $0.6 \%$ agarose base layer. Each condition was plated in triplicates. After 2-3 weeks, images were taken and quantification was performed. Statistical analysis was done using Student's t-test.

\section{Migration and invasion assays}

Trans-well Matrigel invasion assay is an in-vitro method to assess the ability of cells to degrade extracellular matrix proteins in response to a stimulus. Migration of cancer cells was assayed in 24 well Boyden chamber with $8 \mu \mathrm{m}$ pore size polycarbonate membrane (BD Biosciences, San Diego, USA). For invasion assay the membranes, precoated with matrigel (a mix of extracellular matrix proteins) were used (BD bioscience, Sandiego, USA). The matrigel layer serves as a reconstituted basement membrane which occludes the pores. Cells $\left(5 \times 10^{4}\right)$ were resuspended in $500 \mu \mathrm{l}$ serum-free medium and placed in the upper chamber, and the lower chamber was filled with $600 \mu \mathrm{l}$ medium with 10\% FBS (serving as a chemoattractant). Cells were incubated for 22 hours and after incubation, the cells remaining on the upper surface of the membrane were removed by wiping with a wet cotton bud. The cells on the lower surface of the membrane were fixed in chilled methanol and stained with $0.05 \%$ crystal violet and counted under the light microscope. The statistical significance was calculated by student's t-test.

\section{Cell cycle analysis by Flow cytometry}

For analysing the distribution of cells in different phases of cell cycle both the floating and adherent cells were used. The adherent cells were washed with PBS and harvested by trypsinization. The single cell suspension was pelleted down and resuspended in $300 \mu$ of PBS and fixed with chilled $100 \%$ ethanol $(700 \mu l)$ by adding drop by drop while gentle vertexing. Cells were fixed by incubating them in $-20^{\circ} \mathrm{C}$ overnight. Incubation was followed by re-pelleting of cells and complete removal of ethanol by two PBS washes and finally followed by treatment with RNaseA $(10 \mu \mathrm{g} / \mathrm{ml})$ for $2-3 \mathrm{~h}$ at $37^{\circ} \mathrm{C}$. Cells were stained with propidium iodide (PI), $10 \mu \mathrm{g} / \mathrm{ml}$ and subjected to flow cytometry using FACSVERSE instrument (BD Biosciences) to assay the effects on cell cycle profile.

\section{Annexin V-FITC (Fluorescein isothiocyanate) and PI staining}

Annexin V-FITC/PI double staining was utilized to quantify apoptosis in each condition. The apoptotic cells were determined under different conditions by flow cytometry based analyses using Annexin V-FITC Apoptosis kit from Biovision (K-101) following the manufacturer's instructions. The percentage of healthy population, early/late apoptotic population and necrotic population was determined from each condition and percentage of apoptotic cells was plotted.

\section{GSCs and DGCs}


MGG8, MGG6, MGG4, and 1035 GSCs were cultured as neurospheres in Neurobasal medium (Invitrogen, \#21103049) supplemented with EGF (20 ng/ml; R\&D systems, \#236-EG-200), bFGF (20 ng/ml, Peprotech,

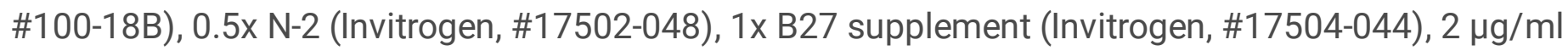
Heparin (Sigma, \#H3149) in low-attachment dishes. For differentiation of GSCs to get DGCs, GSCs were transferred to $10 \%$ serum containing DMEM media in regular adherent plates and cultured for 7 days.

\section{Neurosphere assay and Limiting dilution assay}

GSC cells were infected with lentivirus expressing either NTshRNA or shRNA against PTGERN or ASTN1. After $48 \mathrm{~h}$ of infection, the sphere aggregates formed were dissociated into single cells, counted and plated $10^{4}$ cells/well in ultra-low attachment 6 well dish and cultured for 7 days. Fresh medium was replenished every 2-3 days. Number of spheres were counted after 7 days of plating and plotted graphs.

For limiting dilution assay, neurospheres were dissociated into single cells and counted. Cells were plated in ultra-low attachment 96 well plates wherein a range of cells $(1,10,25,50,75$, and 100 cells/well) were plated into 8 wells for each condition. Fresh medium was replenished every 2-3 days. After 7-10 days, wells forming the spheres were counted in control knockdown and gene knockdown condition. Graph was plotted using ELDA (Extreme Limiting Dilution Analysis) software.

\section{Results}

\section{Regulated CAMs in GBM}

To elucidate the deregulation of CAMs in GBM, we manually curated a comprehensive list of 518 CAMs (Supplementary Table 1) from various sources such as gene Entrez query 'CAMs and homo sapiens' and other related publications in the literature $[20,21]$. We performed an integrated bioinformatics analysis to find out the deregulated CAMs in GBM and the reasons for their deregulation (Supplementary Fig. 1). To identify the transcriptional changes in CAMs in GBM, we analysed the expression of CAMs in TCGA dataset $(n=582)$ and found a total of 181 CAMs to be differentially regulated in GBM as compared to control samples. Among the deregulated CAMs, 88 were significantly upregulated and 93 were significantly downregulated (Fig. 1A). We found nearly an equal percentage ( $48 \%$ and $52 \%$ ) of CAMs differentially expressed in GBM as compared to control. We observed similar results in the REMBRANDT dataset (Supplementary Fig. 2A) and this was also validated in GSE22866 and GSE7696 datasets. We observed that $40-79 \%$ of 181 CAMs to be similarly differentially regulated in these datasets

(Supplementary Fig. 2B, 2C, and Supplementary Table 2). Thus, we identified differentially regulated CAMs in GBM indicating that CAMs might be playing both oncogenic and tumor suppressor functions in GBM.

We examined the possible mechanisms behind the differential regulation of CAMs in GBM. The copy number variation analysis in TCGA data showed that out of the 88 upregulated CAMs, 5 were amplified and among 93 downregulated CAMs, 7 were deleted in more than $1 \%$ of tumors (Supplementary Fig. 3A and Supplementary Table 3 ). Thus, $5.68 \%$ of upregulated CAMs were found to be amplified, whereas 
7.52\% of downregulated CAMs were found to be deleted at their gene loci. Further, we examined the role of epigenetic mechanisms that might lead to the differential regulation of CAMs. Analysis of differential methylation using TCGA methylation data uncovered that out of the 181 deregulated CAMs, 32 CAMs showed differential methylation in GBM as compared to control. We identified that 12 genes are upregulated corresponding to the 18 hypomethylated $\mathrm{CpGs}$ and 20 genes are downregulated corresponding to 28 hypermethylated CpGs (Supplementary Fig. 3B and Supplementary Table 4). This was also validated in our patient cohort (GSE79122) and GSE60274 wherein most of the genes were similarly differentially methylated (Supplementary Fig. 3C). Therefore, we identified $6.6 \%$ of upregulated CAMs to be hypomethylated and $11 \%$ of downregulated CAMs to be hypermethylated.

It is well established that the gene transcript levels can be regulated by miRNAs. We investigated to identify the downregulated miRNAs that are predicted to target upregulated CAMs and upregulated miRNA that are predicted to target downregulated CAMs. We used TCGA expression data for miRNA and mRNA and identified 9 downregulated miRNAs can putatively target 52 upregulated CAMs, and 5 upregulated miRNAs can putatively target 43 downregulated CAMs in GBM (Supplementary Fig. 3D and Supplementary Table 5). Thus, $59 \%$ of the upregulated CAMs and $46.2 \%$ of the downregulated CAMs were regulated by miRNAs suggesting that miRNAs play a pivotal role in the regulation of differentially expressed CAMs apart from copy number variation and DNA methylation (Supplementary Fig. 3E).

\section{Prostaglandin F2 receptor inhibitor (PTGFRN) is upregulated in GBM and is a poor prognostic indicator}

Towards predicting the prognostic values of CAMs, we carried out univariate cox regression analysis using TCGA dataset. This analysis identified 127 CAMs to be significantly correlated with survival. Among them, 84 CAMs were found to correlate to poor prognosis and 43 CAMs were found to correlate to good prognosis in GBM (Supplementary Table 6). A transmembrane scaffolding protein PTGFRN, which predicted poor prognosis in GBM, was chosen for in-depth investigation due to various following reasons. First, patients with high PTGFRN transcripts had significantly lower median survival compared to those with low PTGFRN transcripts (Fig. 1B). Second, PTGFRN transcript levels were found to be significantly upregulated in GBM as compared to control brain samples in multiple datasets (Fig. 1C). Third, it was observed that PTGFRN expression was found to be significantly upregulated: in classical and mesenchymal subtypes as compared to neural and pro-neural subtypes (Fig. 1D), in wild type IDH1 tumors as compared to mutant IDH1 tumors (Fig. 1E), and in G-CIMP- tumors as compared to G-CIMP+ tumors of GBM (Fig. 1F). It is reported that wild type IDH1 and G-CIMP- subtypes of GBM are more aggressive as compared to their counterparts (21). However, no significant difference in expression of PTGFRN was found between MGMT methylated and unmethylated groups of GBM (Fig. 1G). Further, the transcript level of PTGFRN was found to be upregulated in majority of the glioma cell lines as compared to control brain tissues and SVG (Fig. 1H). The protein levels were found to be varying but distinctly higher in T98G, A172, LN229, U373, and U343 as compared to SVG (Fig. 1I). Thus, the results so far indicate that the PTGFRN is highly upregulated in GBM, associated with wild type IDH1 and G-CIMPGBMs and is a predictor of poor prognosis in GBM. 


\section{PTGFRN regulates cell growth, migration, and invasion in GBM}

To explore the role of PTGFRN in glioma development, silencing studies were carried out in U373, U343, T98G, U251, and U87 glioma cell lines. PTGFRN was silenced using specific short hairpin RNA (shRNA) and the silencing was verified by western blot (Fig. 2A, Supplementary Fig. 4A). Silencing PTGFRN significantly reduced cell proliferation, colony formation, anchorage-independent growth, migration, and invasion in U373 (Fig. 2B-2F), U343, T98G, U251, and U87 glioma cells (Supplementary Fig. 4B-4F). Silencing PTGFRN caused G2/M arrest in U373, whereas, G1 arrest in T98G cells (Fig. 2G, Supplementary Fig. 4G). Further, we found that silencing PTGFRN significantly increased Annexin-V positive cells in U373 and $\mathrm{U} 251$ by $52 \%$ and $37 \%$ of the total cells, respectively (Fig. $2 \mathrm{H}$, Supplementary Fig. $4 \mathrm{H}$ ). We also investigated the role of PTGFRN in the regulation of signaling pathways that are highly dysregulated in GBM such as ERK, AKT, and mTOR. Silencing of PTGFRN in U373 and T98G reduced phospho-AKT, phospho-ERK, phospho-4EBP, and phospho-p70S6 (Fig. 2l). These results collectively suggest that PTGFRN could be playing an essential role in growth, migration, invasion, and tumor growth in GBM.

\section{PTGFRN expression is regulated by promoter DNA methylation and miR-137 in GBM}

The analysis of mechanisms behind the deregulation of CAMs in GBM revealed that PTGFRN might be regulated by methylation and miRNAs. We carried out PTGFRN promoter methylation analysis and found that two $\mathrm{CpG}$ probes predicted to be hypomethylated significantly in GBM as compared to control in TCGA 450K methylation array, GSE60274 methylation dataset (27K array), and GSE79122 methylation dataset (450K array) (Fig. 3A). We performed correlation analysis between PTGFRN transcript level and promoter $\mathrm{CpG}$ methylation in GBM and found a significant negative correlation between expression and methylation (Fig. 3B). Further, miRNA predicting algorithms in miRwalk (http://zmf.umm.uniheidelberg.de/apps/zmf/mirwalk2/) predicted that miR-137 can target 3'UTR of PTGFRN and it was found to be significantly downregulated in GBM as compared to control (Fig. 3C). We performed correlation analysis between expression of PTGFRN and miR-137 and found a significant negative correlation between expression of PTGFRN and miR-137 in GBM (Fig. 3D). It was observed that miR-137 putatively targets two specific sites in 3'UTR of PTGFRN (Fig. 3E). The overexpression of miR-137 dramatically reduced the protein levels of PTGFRN in U373 (Fig. 3F). These results suggest that the promoter hypomethylation and miR-137 downregulation might be responsible for upregulation of PTGFRN in GBM.

\section{PTGFRN is upregulated in glioma stem-like cells (GSCs) and is essential for GSC growth}

In order to dissect the role of CAMs in GSCs, we investigated the expression of CAMs in GSE54791 and GSE46016 datasets, which carry the transcriptome profile of GSCs and their corresponding differentiated glioma cells (DGCs) and neural stem cells (NSCs). Bioinformatics analysis revealed that 14 CAMs were significantly upregulated, and 8 CAMs were significantly downregulated specifically in GSCs when compared to both NSCs and DGCs (Fig. 4A). Since we found PTGFRN to be one of the most upregulated CAMs in GSCs, we analysed its expression in various GSC datasets. We found that PTGFRN transcript levels to be significantly upregulated in GSCs as compared to NSCs (GSE46016) and DGCs (GSE54791) 
(Fig. 4B). Further, we also observed that PTGFRN protein levels found to be more in GSCs such as MGG8, MGG6, MGG4, and 1035 than their corresponding DGCs (Fig. 4C). Silencing PTGFRN in MGG6, MGG8, and U343 significantly reduced neurosphere formation as assessed by neurosphere assay and limiting dilution analysis (Fig. 4D, 4E, 4F, Supplementary Fig. 5A, 5B). From these results, we conclude that PTGFRN is upregulated in GSCs and is essential for GSC growth.

\section{Discussion}

Alteration in CAMs is one of the main reasons for enhanced migration and invasion in GBM (22). In this study, we identified nearly $35 \%$ of CAMs analysed were found to be differentially regulated in GBM. Our results corroborate with previous reports that CAMs exhibit both oncogenic and tumor suppressor functions in $\operatorname{GBM}(24,25)$. Further, we explored the mechanism behind the differential expression of CAMs in GBM. More than $50 \%$ of deregulated CAMs identified to be targeted by miRNA indicating their critical role in the deregulation of CAMs. Some of these miRNA-mRNA pairs have already been reported to play a role in glioma biology (25).

We found that PTGFRN expression to be high in glioma tissues, cell lines, and GSCs and its high expression is correlating with patient poor survival and our results corroborated with previous reports (19, 27). We observed that PTGFRN is required for cell growth, migration, invasion, progression of cell cycle, and evading apoptosis in glioma cells and these results suggest its oncogenic role in glioma. It was reported that PTGFRN plays an essential role in muscle regeneration (14) and cell migration in HEK293T (11) and leukocytes (10). Hence, its high expression could be protecting cells from apoptosis, and thereby promoting growth and migration in GBM. An abnormal activation of PI3K/AKT/mTOR signaling has been implicated in tumor initiation, progression, and therapeutic resistance in glioma (27). We found that silencing PTGFRN reduced ERK, AKT, and mTOR signaling in glioma cell lines. This suggests that PTGFRN could be functioning through transmembrane receptor signaling that further modulate prosurvival and promigratory signaling pathways in cancer (28). Our results were further strengthened by the recent reports wherein PTGFRN was found to be conferring resistance to radiation via PI3K-AKT signaling in GBM (18) and its expression correlated with metastatic capacity in lung cancer (16). We identified PTGFRN expression could be regulated by promoter hypomethylation and downregulation of miR-137 which is predicted to target PTGFRN in GBM. Our results concur with the findings of previous report with respect to miR-137 (29).

\section{Conclusion}

This study offers a comprehensive overview of deregulation of CAMs and probable reasons for their regulation in GBM. This study also uncovers for the first time about the promigratory role and regulation of PTGFRN in GBM apart from its role in cell growth. Studying the role of CAMs in GBM biology may be useful for targeting tumor infiltration and PTGFRN can be used as a therapeutic target to treat invasive GBM. 


\section{Abbreviations}

GBM: Glioblastoma; CAMs: Cell Adhesion Molecules; PTGFRN: Prostaglandin F2 receptor inhibitor; CNV: Copy number variation; GSC: Glioma stem-like cell; DGC: Differentiated glioma cell; NSC: Neural stem cell; shRNA: Short hairpin RNA

\section{Declarations}

\section{Ethics approval and consent to participate}

Non-tumorous control brain tissue samples (N1-N5) obtained during the surgery of intractable epilepsy patients at National Institute of Mental Health and Sciences (NIMHANS), Bangalore, India. The samples were obtained after an informed, written consent from all the patients, prior to the initiation of the study. All methods and experimental protocols adopted in this study are in accordance with the guidelines approved by the institutional biosafety clearance committee of NIMHANS, Bangalore and IISc, Bangalore. In the present study, control brain tissue sample derived RNA was used to measure PTGFRN transcript levels.

\section{Consent for publication}

Not applicable

\section{Availability of data and materials and datasets used}

All the analysed data are included as supplementary files. Materials and other data are available with the first author and corresponding author and will be furnished upon request. We utilized in whole or part of the data from The Cancer Genome Atlas (TCGA) pilot project funded by the NHGRI and NCl. The information about TCGA institutions and investigators that constitute TCGA network can be found at http://cancergenome.nih.gov/. We also used REMBRANT, GSE22866, GSE7696, GSE54791, and GSE46016 datasets in this study.

\section{Competing interests}

Authors have declared no competing interests.

\section{Funding}

IISc and DBT (Govt. of India) provided fellowship to UM and TB, respectively. DBT, DST, and CSIR (Govt. of India) provided the major research grant for conducting the present research work. Infrastructure supported by DST-FIST, DBT, and UGC (Govt. of India). KS is awarded J. C. Bose Fellowship from DST.

\section{Authors' contributions}


KS and UM; conceived the idea, KS; arranged the funding, UM and TB; designed experiments, performed experiments, and analysed data. UM and KS wrote the manuscript and all the authors read and approved the manuscript.

\section{Acknowledgments}

We acknowledge Dr. Vani Santosh from Department of Neuropathology and Dr. Arimappamagan Arivazhagan from Department of Neurosurgery, NIMHANS, Bangalore, India, for providing normal brain tissue samples. We acknowledge Dr. Subba Rao and Dr. Deepak K. Saini (IISc, India) for shRNA constructs. UM acknowledges IISc for fellowship. TB acknowledges DBT (Govt. of India) for financial aid. KS acknowledges DBT, DST and CSIR (Govt. of India) for the major research grant. Infrastructure supported by DST-FIST, DBT, and UGC. KS is awarded J. C. Bose Fellowship from DST.

\section{References}

1. R. Stupp, et al., Radiotherapy plus concomitant and adjuvant temozolomide for globlastoma, N. Engl. J. Med. 352 (2005) 987-996.

2. F. B. Furnari, et al., Malignant astrocytic glioma: genetics, biology, and paths to treatment, Genes Dev. 21 (21) (2007) 2683-2710.

3. A. V. E. T. Brno, Adhesion molecules in biology and oncology, Pathology (2004) 93-104.

4. Y. Huang, R. Baluna, and E. S. Vitetta, Adhesion molecules as targets for cancer therapy, Histol. Histopathol. 12 (1997) 467-477.

5. J. D. Lathia, et al., Integrin alpha 6 regulates glioblastoma stem cells, Cell Stem Cell 6 (5) (2010) 421-432.

6. A. Goenaga, et al., Identification and characterization of tumor antigens by using antibody phage display and intrabody strategies, Molecular Immunology 44 (2007) 3777-3788.

7. M. He, et al., Hepatocellular carcinoma-derived exosomes promote motility of immortalized hepatocyte through transfer of oncogenic proteins and RNAs, Carcinogenesis 36 (9) (2015) 10081018.

8. T. V. Kolesnikova, et al., Glioblastoma inhibition by cell surface immunoglobulin protein, Neoplasia 11 (1) (2009) 77-86.

9. Y. Wu, et al., Transcriptome profiling of the cancer, adjacent non-tumor and distant normal tissues from a colorectal cancer catient by deep sequencing, PloS One 7 (8) (2012) e41001.

10. J. George, et al., Comprehensive genomic profiles of small cell lung cancer, Nature 524 (2015) 4753.

11. S. Charrin, et al., The major CD9 and CD81 molecular partner. Identification and characterization of the complexes, J. Biol. Chem. 276 (17) (2001)14329-14337.

12. C. Chambrion and Francois Le. Naour, The tetraspanins CD9 and CD81 regulate CD9P1-induced effects on cell migration, PloS One 5 (6) (2010) e11219. 
13. M. Sala-Valdés, et al., EWI-2 and EWI-F link the tetraspanin web to the actin cytoskeleton through their direct association with Ezrin-Radixin-Moesin proteins, J. Biol. Chem. 281 (28) (2006) 1966519675.

14. T. Wakabayashi, et al., Analysis of the $y$-secretase interactome and validation of its association with tetraspanin-enriched microdomains, Nat. Cell Biol. 11(11) (2011) 1340-1346.

15. D. J. Orlicky, et al., Synthesis and accumulation of a receptor regulatory protein associated with lipid droplet accumulation in 3T3-L1 cells, J. Lipid Res. 39 (1998) 1152-1161.

16. S. Charrin, et al., Normal muscle regeneration requires tight control of muscle cell fusion by tetraspanins CD9 and CD81, Nat. Commun. 41674 (2013).

17. W. Guilmain, et al., CD9P-1 expression correlates with the metastatic status of lung cancer, and a truncated form of CD9P-1, GS-168AT2, inhibits in vivo tumour growth, Br. J. Cancer 4 (3) (2011) 496504.

18. S. Charrin, et al., The Ig domain protein CD9P-1 down-regulates CD81 ability to support Plasmodium yoelii infection, J. Biol. Chem. 284 (46) (2009) 31572-31578.

19. B. Aguila, et al., The Ig superfamily protein PTGFRN coordinates survival signaling in glioblastoma multiforme, Cancer Lett. 462 (2019) 33-42.

20. C. Y. Li, et al., OKCAM: An ontology-based, human-centered knowledgebase for cell adhesion molecules, Nucleic Acids Res. 37 (2009) 251-260.

21. X. Zhong, et al., Human cell adhesion molecules: Annotated functional subtypes and overrepresentation of addiction-associated genes, Ann. N. Y. Acad. Sci. 1349 (1) (2015) 83-95.

22. S. M. Turaga and J. D. Lathia, Adhering towards tumorigenicity: altered adhesion mechanisms in glioblastoma cancer stem cells, CNS Oncol. 5 (4) (2016) 251-259.

23. L. Malric, et al., Interest of integrins targeting in glioblastoma according to tumor heterogeneity and cancer stem cell paradigm: an update, Oncotarget 8 (49) (2017) 86947-86968.

24. Y. H. Bai, et al., A novel tumor-suppressor, $\mathrm{CDH} 18$, inhibits glioma cell invasiveness via UQCRC2 and correlates with the prognosis of glioma patients, Cell Physiol. Biochem. 48 (4) (2018) 1755-1770.

25. Y. S. Liu, et al., MIR-181b modulates EGFR-dependent VCAM-1 expression and monocyte adhesion in glioblastoma, Oncogene 36 (35) (2017) 5006-5022.

26. D. Ghosh, et al., A cell-surface membrane protein signature for glioblastoma, Cell Syst. 4 (5) (2017) 516-529.

27. X. Li, et al., PI3K/Akt/mTOR signaling pathway and targeted therapy for glioblastoma, Oncotarget 7 (22) (2016) 33440-33450.

28. J. F. Hastings, et al., The extracellular matrix as a key regulator of intracellular signaling networks, $\mathrm{Br}$. J. Pharmacol. 176 (1) (2019) 82-92.

29. S. Tamim, et al., Genomic analyses reveal broad impact of miR-137 on genes associated with malignant transformation and neuronal differentiation in glioblastoma cells, PLoS One (1) (2014) e855919. 
A



B

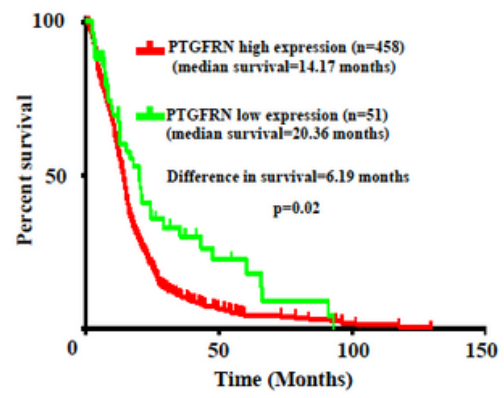

C



Figure 1; Mala et al., 2021

D

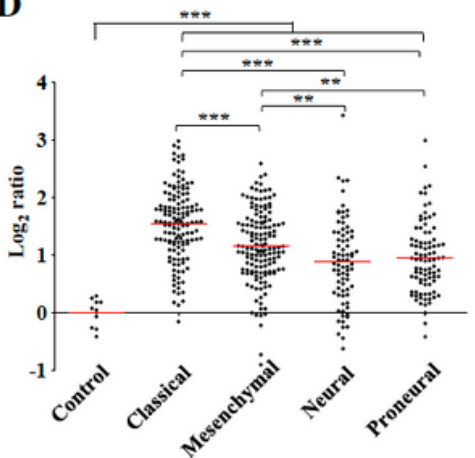

E



F



G

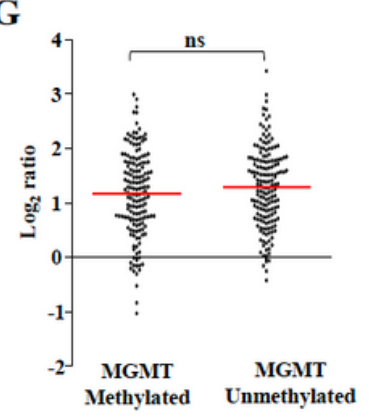

H



I

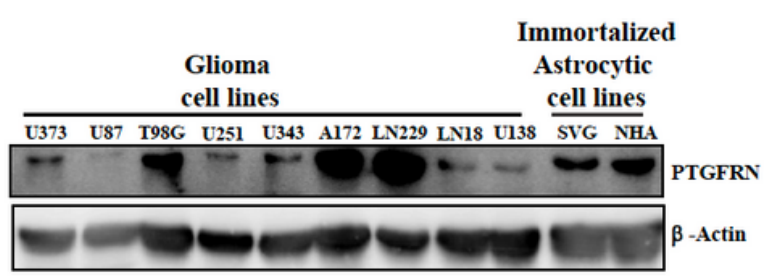

\section{Figure 1}

Transcriptional aberrations identified in CAMs in GBM predict the pro-tumorigenic potential of PTGFRN in GBM. (A) Volcano graph depicting upregulated (red), downregulated (green), and unregulated (black) CAMs in GBM samples ( $n=572)$ as compared to control samples $(n=10)$. The horizontal line separates the CAMs having a significant difference in expression ( $p$-value $\leq 0.05$ ). Vertical lines show the cut off value $\leq-0.58$ or $\geq 0.58 \log 2$ ratio for classifying differentially regulated CAMs. (B) Kaplan Meier curve shows overall survival difference between PTGFRN-high and low transcript groups of GBM. (C) Scatter plots show the transcript level of PTGFRN in GBM in TCGA Agilent, TCGA RNA-Seq, REMBRANDT, and in TCGA GBM subtypes: (D) classical, mesenchymal, neural, and proneural, (E) mut-IDH1 and wt-IDH1, (F) GCIMP+ and G-CIMP-, and (G) MGMT methylated and MGMT unmethylated groups. (H) Bar graph shows transcript level of PTGFRN in glioma cell lines and immortalized human astrocytes (SVG and NHA), and control brain samples as measured by RT-qPCR. (I) Immunoblot shows protein level of PTGFRN in glioma cell lines and immortalized astrocytes and $\beta$-Actin served as loading control (required portion of the blot 
is shown after cropping from the whole blot). The significance was tested using Mann-Whitney t-test and the symbols are indicated. (ns) not significant; (*) $p \leq 0.05 ;(* *) p \leq 0.01$, and (***) $p \leq 0.001$.
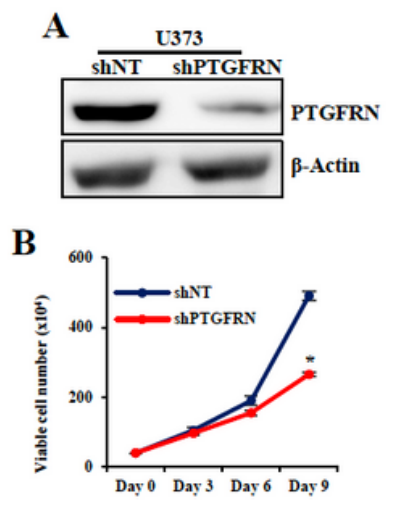

G
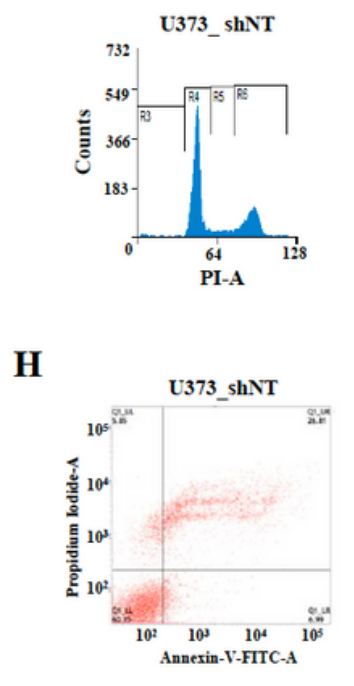

C
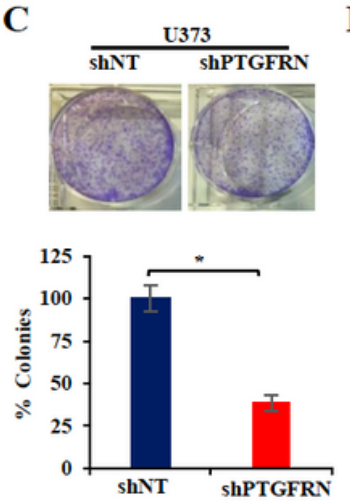

D
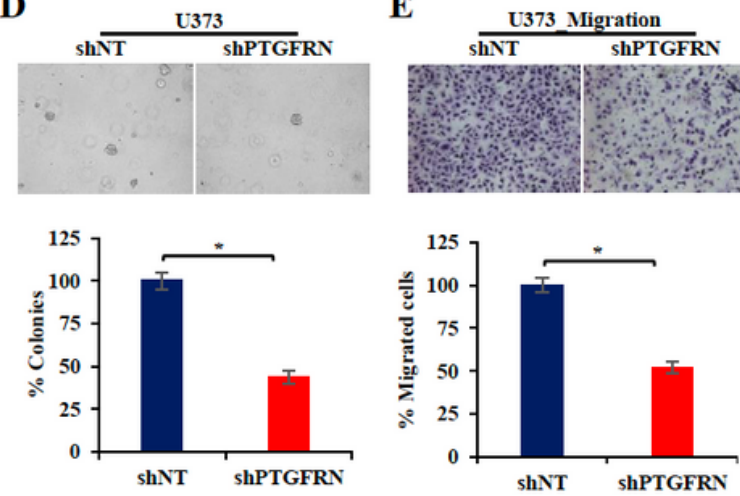

E

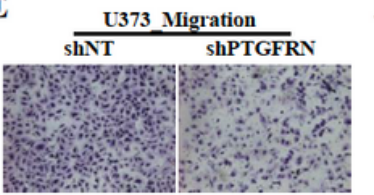

Figure 2; Mala et al., 2021

F
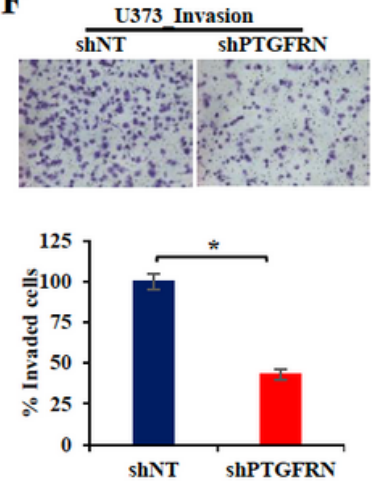

I



Figure 2

Knockdown of PTGFRN diminishes cell growth, migration, and invasion in GBM In U373 cells PTGFRN silenced with either shPTGFRN or shNT, (A) immunoblot shows protein levels of PTGFRN and $\beta$-Actin served as loading control (required portion of the blot is shown after cropping from the whole blot), (B) line graph shows the relative cell viability. Representative images show (C) colony number, (D) soft agar colony number, (E) migration, and (F) invasion after silencing PTGFRN in U373 and quantification shown as bar graphs. (G) Histograms represent the DNA content (stained with $\mathrm{PI}$ ) and $(\mathrm{H})$ flow cytometry dot plots represent the Annexin-V positive cells in control and PTGFRN silenced cells in U373 and quantification shown as bar graph. (I) Immunoblots show the protein levels of PTGFRN, p-ERK, ERK, pAKT, AKT, p-4EBP, 4EBP, p-p70S6, and p70S6 after silencing PTGFRN in U373 and T98G. $\beta$-Actin used as loading control in western blotting (required portion of the blot is shown after cropping from the whole blot). The quantification for each blot given below the blot. The t-test was tested using Student's t-test and the symbols are indicated. (ns) not significant; $(*) p \leq 0.05 ;(* *) p \leq 0.01$ and (***) $p \leq 0.001$. 
$\mathbf{A}$
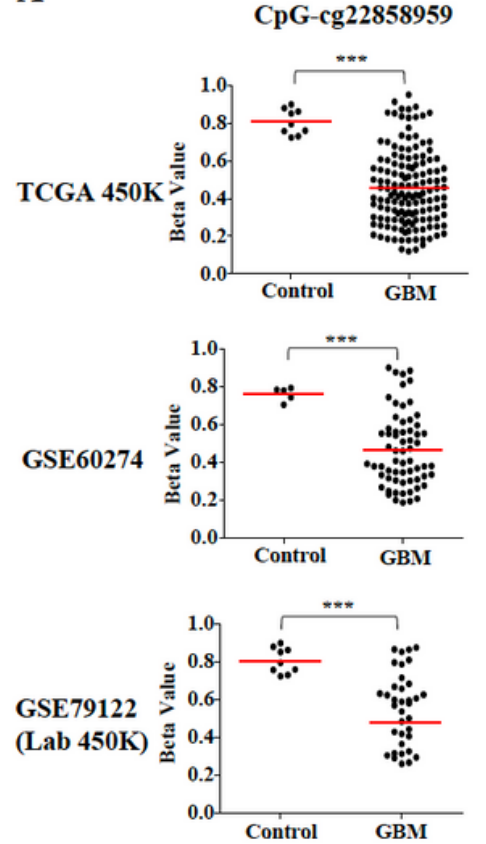

B

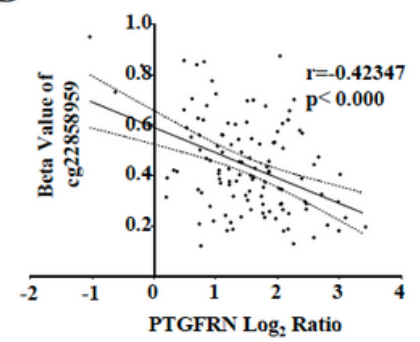

CpG-cg22448232


C



D

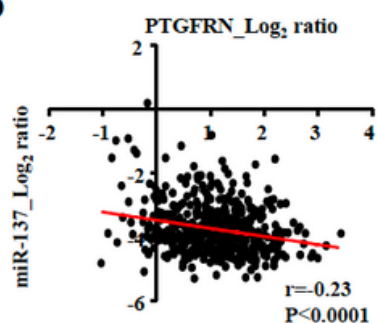

$\mathbf{E}$

3' UTR of PTGFRN_3383 bp



$\mathbf{F}$



Figure 3

Regulation of PTGFRN by promoter methylation and miR-137 (A) Scatter plots depicting the beta values for CpGs cg22858959 and cg22448232 in control and GBM samples in TCGA 450K, GSE60274, and GSE79122 datasets. (B) Correlation graphs show the correlation between $\mathrm{CpG}$ methylation and expression of PTGFRN in TCGA GBM samples. (C) Scatter plot depicting the transcript levels of miR-137 in control and GBM samples in TCGA dataset. (D) Dot plot represent the correlation between expression of PTGFRN and miR-137 in TCGA GBM samples. (E) Schematic shows miR-137 targeting sites on the 3'UTR of PTGFRN and base pairing between miR-137 and targeted sequence on 3'UTR. (F) Immunoblot shows PTGFRN protein levels in vector and miR-137 overexpression in U373 and $\beta$-Actin was used as loading control (required portion of the blot is shown after cropping from the whole blot). The significance was performed using Mann-Whitney t-test and the symbols are indicated, (ns) not significant; $(*) p \leq 0.05 ;(* *) p \leq 0.01$ and $(* * *) p \leq 0.001$. 
A

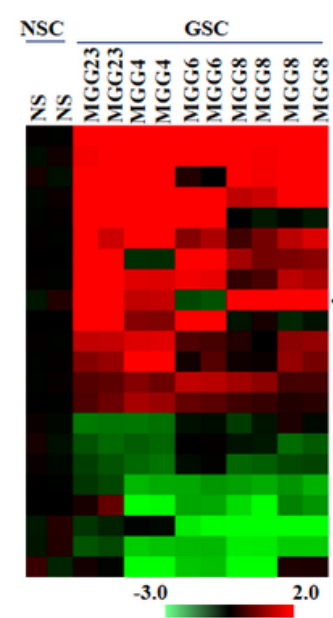



DGC

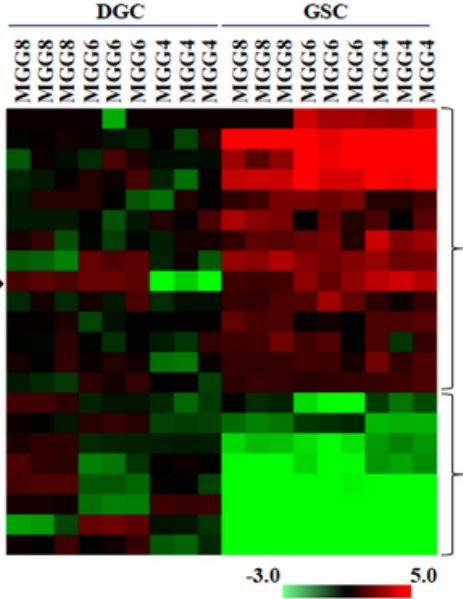

$\mathbf{E}$
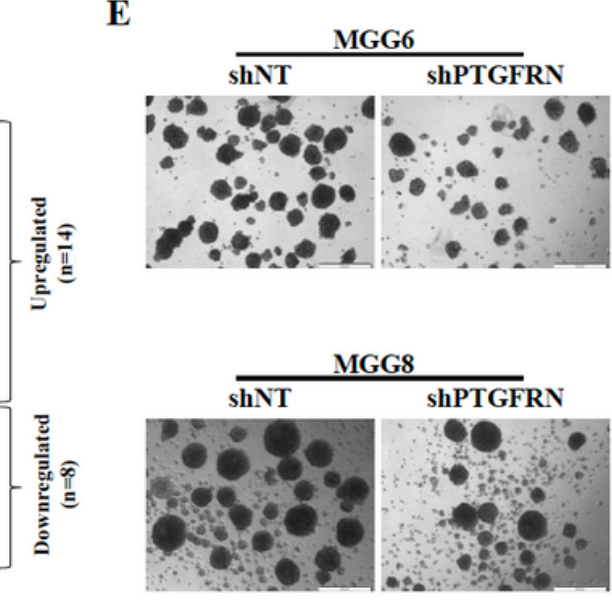

B

GSE119834

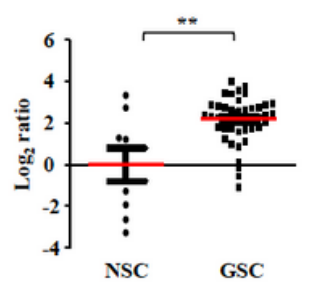

C

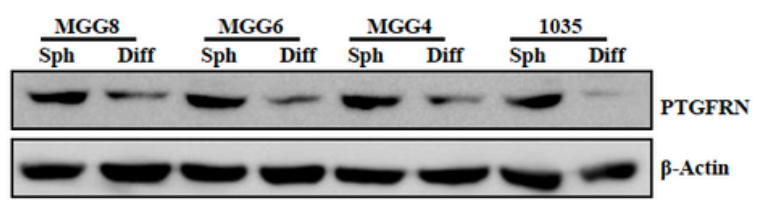

GSE31262

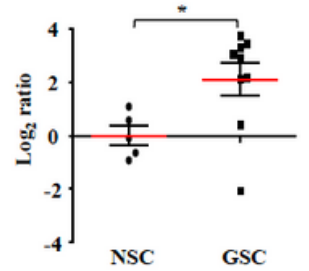

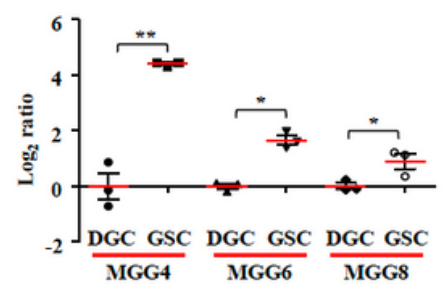

D

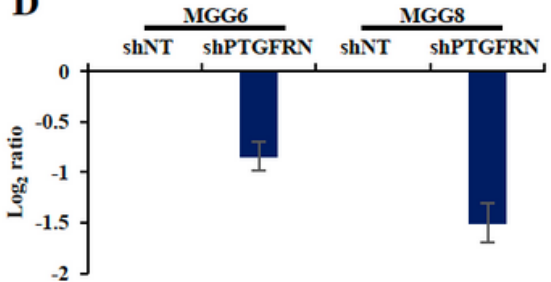

GSE54791
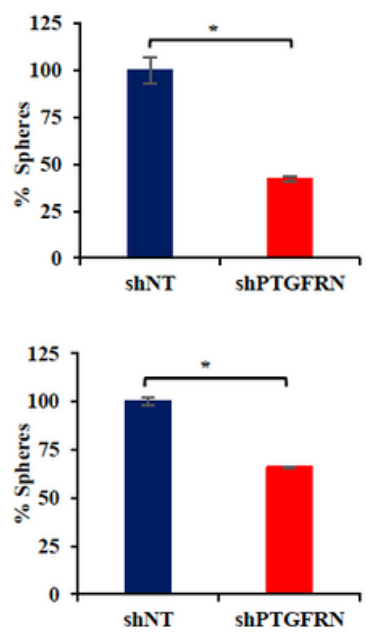

F
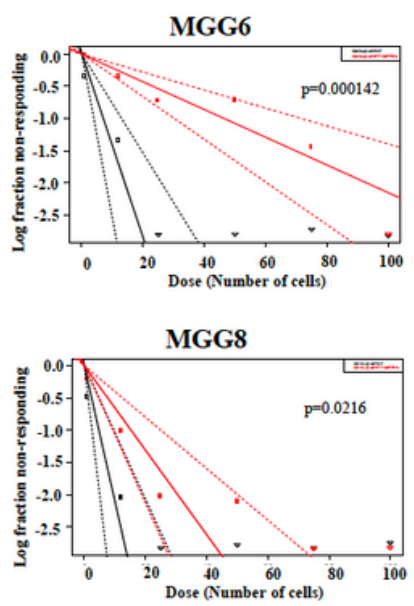

Figure 4

PTGFRN is upregulated in GSCs and required for its growth A) Heatmaps representing the differentially expressed CAMs in GSCs as compared to NSCs (left) and in GSCs as compared to DGCs (right). GSE46016 (GSC vs NSC, gene microarray) and GSE54791 (GSC vs DGC, RNA-Seq) datasets were used for the analysis. Red and green color indicates the upregulated and downregulated CAMs, respectively. (B) Scatter plots represent the transcript levels of PTGFRN in different datasets GSE54791, GSE119834, and GSE31262. (C) Immunoblot shows the protein levels of PTGFRN in GSCs and corresponding DGCs in MGG8, MGG6, MGG4, and 1035 and $\beta$-Actin was used as loading control (required portion of the blot is shown after cropping from the whole blot). GSCs cultured as neurospheres, indicated as Sph (Spheres) and DGCs cultured as monolayer and indicated as Diff (Differentiated cells). (D) Bar graph shows the transcript levels of PTGFRN in shNT and shPTGFRN in MGG6 and MGG8. (E) Representative images of neurospheres and their quantification and $(F)$ Line graphs show the limiting dilution analysis in MGG6 and MGG8 after silencing PTGFRN. Student's t-test was performed to test the statistical significance and the symbols are shown. (ns) not significant; $(*) p \leq 05 ;(* \star) p \leq 0.01$ and $(* \star *) p \leq 0.001$. 


\section{Supplementary Files}

This is a list of supplementary files associated with this preprint. Click to download.

- AdditionalFileRawblotsCAMsandPTGFRNinGliomaBMCCancer.pdf

- Malaetal.SupplementaryTables16.xIsx

- Supple.Fig.1.pdf

- Supple.Fig.2.pdf

- Supple.Fig.3.pdf

- Supple.Fig.4.pdf

- Supple.Fig.5.pdf 